\title{
The Wheel - Vehicle Considered as a Material Point: An Approximate Theory of Automobiles
}

\author{
Thanassis Dialynas \\ Department of Physics, University of Crete, Heraklion, Greece
}

Email address:

tedialynas@gmail.com

\section{To cite this article:}

Thanassis Dialynas. The Wheel -Vehicle Considered as a Material Point: An Approximate Theory of Automobiles. American Journal of Physics and Applications. Vol. 9, No. 2, 2021, pp. 34-41. doi: 10.11648/j.ajpa.20210902.12

Received: February 2, 2021; Accepted: February 24, 2021; Published: April 26, 2021

\begin{abstract}
The objective of the article is the mathematical description of the car motion in the most possible general form using Newton's second law and the forces that act on it when they are known. In the first section, the forces that act on the vehicle are described and the normal (usual) conditions of driving are considered. Secondly the dynamical equation of motion baced on Newton's second law is introduced which is in general a non-linear second order ordinary differential equation. Various cases are discussed such as going uphill, downhill, accelerating, decelerating etc. In Section 3, the energy consumption of the fuel is discussed and it is stated that it is consisted of two parts this of the "idle" worke and this of the sustainment of the motion. Besides it is shown that for a certain space " $\mathrm{s}$ " there is one unique speed that minimizes the consumption of fuel. In Section 4, the basic "defect" of the equation of motion which is the inclusion in the equation of the unknown driving force $F(t)$ it is shown that it can be "circumvented" with energetic considerations leading to an equation having at the right - hand side the speed in the denominator and the excess revolutions per minute in the numerator. The resulting equation is such that a knowledge of $\delta_{\mathrm{r}}(\mathrm{t})=(\mathrm{rpm})(\mathrm{t})-(\mathrm{rpm})_{0}$ can, by the numerical solution of the equation, lead to the function of speed and so a relation is established detween the velocity $(\mathrm{u}(\mathrm{t}))$ and the excess $(\mathrm{rpm})$ which can be cheched as true or false by the aposteriori resister of the tachograph $(\mathrm{u}(\mathrm{t}))$ and rotation - counter $(\mathrm{rpm}(\mathrm{t}))$. Finally, in Section 5, we calculate, using the decelerating motion of a car in a flat road (when somebody leaves the throttle) all the kinematical and "energetical" constants that are introduced in the previous sections for sixth gear such as $\mathrm{F}_{\mathrm{c}, 6}, \mathrm{~b}_{6}, \sigma_{6}, \lambda_{6}$ which can be used, post - hoc, to examine together with $\delta_{\mathrm{r}}(\mathrm{t})$ if the real velocityof a vehicle coincides with the prediction that a computer can make. Besides for a flat road, the power of a car can be estimated for instance when it has $\mathrm{u}=120 \mathrm{~km} / \mathrm{h}$ at $\mathrm{rpm}=3000$ and in the $6^{\text {th }}$ gear, giving for power $-45 \mathrm{HP}$ which is a very reasonable estimate in order of magnitude.
\end{abstract}

Keywords: Motion of a Vehicle, Newton's Second Law, Ordinary Differential Eqs

\section{Introduction}

In the last three decades, there is a considerable effort of physicists for understanding the physics of automobiles and related topics [5-20].

The basic tool is Newton's Second Law which if it is combined with other considerations like the theory of o.d.e.'s can probably go far, giving a theory of automobile considered as a "material point".

A motion of a car is a motion of certain trajectory (for instance a closed road) which may be flat, may have turns or may pass through hills (uphill or downhill).

If we impose all the forces that are exert on the car (considered as a material point) it is a matter of elementary differential geometry of a certain trajectory, to see that all the forces not tangentional to the road are used to keep the car in the certain trajectory, letting the tangential forces, to determine the velocity (speed) of the "material point" [1-4].

The tangentional forces are:

1. The forward force of the engine $F(t)$

2. The friction - resistance of the air denoted by $\mathrm{R}(|\mathrm{u}|) \hat{u}=-\left(F_{c}+b_{1}|\mathrm{u}|+b_{2}|u|^{2}+\ldots\right) \hat{u}$, which for small velocities (speeds) is equivalent to $-F_{c} \hat{u}-\mathrm{bu}=-F_{c}-\mathrm{bu}$ for $\mathrm{u} \geq 0$

3. The component of the weight which is tangentional to the road.

The above considerations are equivalent (for "normal motion") to a trajectory which is found to be at a plane 
perpendicular to the horizon and a trajectory that at most goes up and down if the "original" trajectory passes through hills.

[The two trajectories, the "original" and the "equivalent" predict the same tangentional velocity $\left.\mathrm{u}(\mathrm{t}) \geq 0, \mathrm{u}(\mathrm{t})=\frac{d s}{d t}\right]$.

Since $u(t) \geq 0$ we have only forward motion i.e. reverse velocity is excluded from our considerations.

The above discussion is valid only under "normal conditions of driving" by which we mean:

1. No sliding or spinning of the wheels

2. The forces exerted on the car by the "environment" (as a reaction) are such to keep the vehicle on the trajectory of the road

3. The power of the engine is such that to keep the vehicle in constant or accelerating motion without loosing a part of it. (For instance in a very abrupt uphill road)

4. No throttle (gas pedal) and brake can be pushed at the same time.

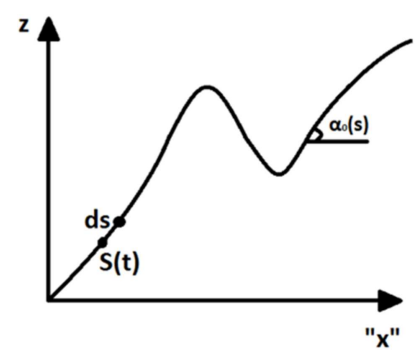

Figure 1. The equivalent trajectory.

\section{Dynamical Equations}

As we have explained in the introduction, for the motion in a $3-\mathrm{D}$ curve there is an equivalence between the original trajectory and the equivalent trajectory regarding the tangential motion and the upgoing in a hill or downgoing afterwards:

The fundamental general equations that describe the motion is:

$$
\begin{gathered}
\mathrm{m} \frac{d u}{d t}=-F_{c} \theta(\mathrm{u})-\mathrm{bu}+\mathrm{F}(t)-\operatorname{mgsin}\left[\alpha_{0}(s)\right] \\
\mathrm{u}=\frac{d s}{d t} \geq 0 \text { and } \mathrm{F}(t) \geq 0
\end{gathered}
$$

Where

1. $F_{c}$ is the constant friction exerted to the vehicle when it moves,

2. - bu is the air resistance,

3. $\mathrm{F}(t)$ is the engine force and

4. Mgsin $\left[\alpha_{0}(s)\right]$ is the component of the weight which is exerted reversely to the motion if $\left(0 \leq \alpha_{0}<\frac{\pi}{2}\right)$ or in alignment to the motion, if $\left(-\frac{\pi}{2}<\alpha_{0} \leq 0\right)$.

Note that the function $\alpha_{0}(\mathrm{~s})$ is a "known" characteristic of the "constant" trajectory and not of the motion which we do not know still. ( $\mathrm{s}=$ the length of the trajectory). Furthermore $F(t)$ is the force of the engine and the function $\theta(u)$ is $\theta(\mathrm{u})=\left\{\begin{array}{l}1 \mathrm{u}>0 \\ 0 \mathrm{u} \leq 0\end{array}\right\}$.

The above equation is too general and therefore it is "useless"
(!), since it is very difficult to come into conclusions [1 - 4].

A simplified version of the above equations for a constant inclination of the road and considering $\mathrm{F}(t) \geq 0, F_{c g} \geq 0$, $\overline{\mathrm{F}}(t) \geq 0$ is the following:

$$
\mathrm{m} \frac{d u}{d t}+\mathrm{bu}=\overline{\mathrm{F}}(t)=\mathrm{F}(t)-F_{c g}
$$

where:

$$
F_{c g}=\left\{\begin{array}{c}
F_{c} \text { (motion in horizontal level) } \\
F_{c}+\operatorname{mgsin} \alpha_{0}(\text { going uphill) } \\
F_{c}-\operatorname{mgsin} \alpha_{0}\left\langle\begin{array}{c}
\text { slightly downhill } \\
F_{c} \geq \operatorname{mg} \text { or } \operatorname{mgsin} \alpha_{0} \leq F_{c}
\end{array}\right.
\end{array}\right.
$$

In all three above cases: $F_{c g}>0$.

For the last case of steep downhill motion we have:

$$
\mathrm{m} \frac{d u}{d t}+\mathrm{bu}=\overline{\mathrm{F}}(t)=\mathrm{F}(t)+F_{g c}
$$

$F_{g c}=\operatorname{mgsin} \alpha_{0}-F_{c}>0$ (steep downhill)

Equations (2) and (3) for "arbitrary" $F(t)$ have the solution [1-4]:

$$
\mathrm{u}(t)=u_{0} e^{-\frac{b}{m} t}+e^{-\frac{b}{m} t} \int_{0}^{t} e^{\frac{b}{m} t^{\prime}} \overline{\mathrm{F}}\left(t^{\prime}\right) d t^{\prime}
$$

Where

$\overline{\mathrm{F}}\left(t^{\prime}\right)$ can be either positive, negative or zero. (in eq. (3) only positive).

In eqs (2) and (3) if the gas petal is not used, then $\mathrm{F}(\mathrm{t})$ will be put to zero $(\mathrm{F}(\mathrm{t})=0)$.

If in addition $\mathrm{t}$ he brake is pushed then $\mathrm{F}(\mathrm{t})=-F_{b}\left(F_{b} \approx\right.$ constant) giving totally

$$
\mathrm{F}(\mathrm{t})=-F_{b}-F_{c g}
$$

or

$$
\mathrm{F}(\mathrm{t})=-F_{b}+F_{g c}
$$

From the general solution (4) one can get for two different instants of the motion $\left[t_{1}, t_{2}\right]$ that

$$
\mathrm{u}\left(t_{2}\right) e^{\frac{b}{m} t_{2}}-\mathrm{u}\left(t_{1}\right) e^{\frac{b}{m} t_{1}}=\int_{t_{1}}^{t_{2}} e^{\frac{b}{m} t} \overline{\mathrm{F}}(\mathrm{t}) d t .
$$

If in addition $\overline{\mathrm{F}}(\mathrm{t}) \leq 0$ for $\mathrm{t}$ in $\left[t_{1}, t_{2}\right]\left(0 \leq \mathrm{F}(\mathrm{t}) \leq F_{c g}\right)$ then it is easy to prove that $\mathrm{u}\left(t_{2}\right)<\mathrm{u}\left(t_{1}\right)$ given that $t_{2}>t_{1}$. On the contrary if $\overline{\mathrm{F}}(\mathrm{t}) \geq 0$ for $\mathrm{t}$ in $\left[t_{1}, t_{2}\right]$ it is not easy (if impossible) to prove that $\mathrm{u}\left(t_{2}\right)>\mathrm{u}\left(t_{1}\right)$. In the first case we have deceleration and in the second probably acceleration.

The situation is the following:

Suppose one drives a car in an horizontal level for instance without a gravitational force but subject to the forces $-F_{c}$ (friction) and - bu (air resistance) [equation (2)] then as soon as one leaves the throttle, he will immediately feel the deceleration exerted on the wheels and subsequently on the car. The opposite situation happens when one pushes the throttle more, in which case one feels the acceleration, not immediately but after a few seconds (or fractions of seconds).

But how a car is initiating its motion, up to now we have considered only the first gear in eqs (1), (2), (3)?. Actually 
these equations have to be replaced by other that contain the costants $b_{g} \Leftrightarrow \mathrm{b}, F_{c g} \Leftrightarrow F_{c g, g}$ and probably $\mathrm{F}(\mathrm{t}) \Leftrightarrow F_{g}(t)$ where the index $g$ (not to be confused with the $g$ of gravity which is also used) denotes the gear, for instance $g$ belongs $\{1,2,3,4,5,6\}$.

In other words a car is not unique but for instance 6 in our case, one for each gear. Automatic cars probably need a different manipulation which has to take into account the mechanism of changing gears automatically.

The initiation of motion in a gear - car is simple if the car is found to be in a steep downhill road since $F_{g c}>0$ and $\mathrm{F}(\mathrm{t})$ $\geq 0$ (eq. 3). The start - up does not give any problems.

However, in the case of (eq. 4) the "combined" driving force $\overline{\mathrm{F}}(\mathrm{t})=\mathrm{F}(\mathrm{t})-F_{c g}$, which may well be negative in which case the vehicle can not start the motion.

$$
\mathrm{u}(\mathrm{t})=e^{-\frac{b}{m} t} \int_{0}^{t} e^{\frac{b}{m} t^{\prime}} \overline{\mathrm{F}}\left(\mathrm{t}^{\prime}\right) d t^{\prime}, \mathrm{u}(\mathrm{t}) \geq 0
$$

It is obvious that at $\mathrm{t}=0$ the motion can not be initated unless, $\mathrm{F}(0)>F_{c g}$.

For subsequent times, it's not necessary to have $\mathrm{F}(\mathrm{t}) \geq F_{c g}$ for $t \geq 0$ in order the motion to be retained as shows the following diagramm.

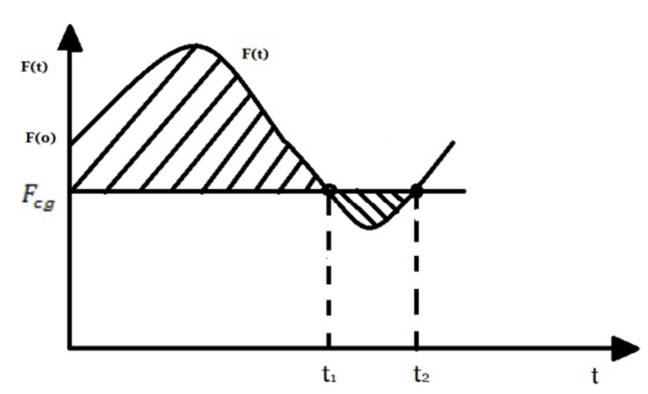

Figure 2. The function $F(t)$ with respect to constant $F_{c g}$.

Defining

$$
\begin{gathered}
I_{F}=\int_{t_{1}}^{t_{2}} \overline{\mathrm{F}}\left(\mathrm{t}^{\prime}\right) d t^{\prime}=I_{\bar{F}}\left(t_{1}, t_{2}\right) \\
I_{b}=\int_{t_{1}}^{t_{2}} e^{\frac{b}{m} t^{\prime}} \overline{\mathrm{F}}\left(\mathrm{t}^{\prime}\right) d t^{\prime}=I_{b}\left(t_{1}, t_{2}\right)
\end{gathered}
$$

If $\overline{\mathrm{F}}(\mathrm{t}) \leq 0$ for $\mathrm{t}$ in $\left[t_{1}, t_{2}\right] \rightarrow I_{F}<0 \rightarrow I_{b}<0$

The inverse is not necessarly true. Therefore the sign of $I_{b}$ is determined by the sign of $I_{F}$.

Suppose that we have three time intervals $\left[0, t_{1}\right],\left[t_{1}, t_{2}\right]$, $\left[t_{2}, t_{3}\right]$ for which the $\bar{F}$ is positive, negative and positive again. (Figure 2)

If $\mathrm{F}(0)>F_{c g}$, the vehicle moves until $t_{1}$, gets in (positive) speed. From $t_{1}$ to $t_{2}$ the calculation of the correspoding solution for which we have $I_{b}\left[t_{1}, t_{2}\right]<0, I_{F}\left[t_{1}, t_{2}\right]<0$ which means that the speed will be reduced by a certain amount if not again becoming 0 , in which case it cannot start - up again. (see Figure 2)

\section{Equations of Energy - Balance}

Energy minimization for certain space "s"

When the engine of a car "works" but the car is not moving we have the situation of the "idle" work as we say.

In this case where there is no gear in the gear - box and the engine works, all the energy that is given to the pistons (heat excluded) of the machine is used to overcome frictions and resistances as the metal parts react one on the surface of the other. The energy for this process as well as all the energy used for the functions of the car (motion, acceleration, uphill going etc) is given by the fuel but in this last case, energy must be provided in addition to the idle work.

From the above considerations we may write:

$$
\begin{gathered}
\left(\frac{d E}{d t}\right)_{0}=P_{0} \\
\left(\frac{d E}{d t}\right)_{1}=P_{01}+(u F)_{1}
\end{gathered}
$$

In the first equation $\left(\frac{d E}{d t}\right)_{0}$ is the consuption of the energy fuel and $P_{0}$ is a constant power in the "idle" work. In the second equation - which is referd to the $1^{\text {st }}$ gear - it is easy to identify the term $P_{01}$ as the "idle work" of the first gear and the term $\mathrm{u}^{*} \mathrm{~F}$ as the extra power needed to make the vehicle to sustain motion or to accelerate or to go uphill.

For every gear $(\mathrm{g})$ it can always be written

$$
\left(\frac{d E}{d t}\right)_{g}=P_{0 g}+(u F)_{g}
$$

Where $\mathrm{u}(\mathrm{t}) \geq 0$ and $\mathrm{F}(\mathrm{t}) \geq 0$.

Where $F(t)$ is the force excerted to the car by the engine. (actually the wheels excert a force on the environment and the environment excerts a reaction on the car, here denoted by $\mathrm{F}(\mathrm{t}))$.

In equation 9, we usually drop the "index" $\mathrm{g}$ for simplicity and because we are usually interested in kinematic changes, that happen to a car, without, a change in the gear - box.

Usually when Newton's eq. is given together with the "driving force" $(\mathrm{F}(\mathrm{t}))$, then the finding of the kinematic properties is done through the solution of an o.d.e. (i.v.p).

(Ordinary differential eq. - initial value problem). In the case where the driving force is not known, then the solution $(\mathrm{s}(\mathrm{t}), \mathrm{u}(\mathrm{t}))$ cannot be found in full detail. However writing the driving - unknown force as a function of the kinematic terms, conclusions may be infered for other quantities, like energy, momentum, e.t.c.

The two driving forces of the previons sections are

$$
\begin{gathered}
\mathrm{F}(\mathrm{t})=\mathrm{m} \frac{d u}{d t}+\mathrm{bu}+F_{c g}, F_{c g}>0 \\
\mathrm{~F}(\mathrm{t})=\mathrm{m} \frac{d u}{d t}+\mathrm{bu}-F_{g c}, F_{g c}>0
\end{gathered}
$$

When the car moves the quantity

$$
\left(\frac{d E}{d t}\right)=P_{0}+\mathrm{uF}
$$

is the rate of consumption of energy due to the idle work and the sustainment of motion $(\mathrm{uF})$.

After the appropriate substitutions we have

$$
\left(\frac{d E}{d t}\right)=P_{0}+\mathrm{u}\left(\mathrm{ma}+\mathrm{bu} \pm F_{c g}\right)
$$


In the above formulae we have put in the first formula $F_{c g}$ $\rightarrow F_{c g}$ and in the second $F_{g c} \rightarrow F_{c g}$ in order not to write double expressions.

No confusion can be made since the two cases may be discriminated by the signs \pm . For what is $F_{c g}$ and $F_{g c}$ see the energy consumed in a long intervall of the time $\mathrm{T}$, it is appropriate to integrate eq. (13) with respect to time. Doing so, and considering that the speed is kept more or less constant and that the initial acceleration is small for a small interval of time and that the vehicle operates in "normal conditions of driving" we have

$$
\mathrm{E}(\mathrm{T}, \mathrm{u})=P_{0} \mathrm{~T}+\frac{1}{2} \mathrm{~m} u^{2}+\mathrm{b} u^{2} \mathrm{~T} \pm F_{c g} \mathrm{uT}
$$

At this point, it has to be mentioned that the above eq. cannot answer the question in how much time, a vehicle moving with velocity (speed) $u$ will consumme the less energy and subsequently fuel, since thisfunction of energy is an increasing function of time $\mathrm{T}$. Making the substitution $\mathrm{T}=\frac{s}{u}$, we have

$$
\mathrm{E}(\mathrm{s}, \mathrm{u})=P_{0} \frac{s}{u}+\frac{1}{2} \mathrm{~m} u^{2}+\mathrm{bus} \pm F_{c g} \mathrm{~s}
$$

With this formula we can ask the question for minimization of the expression with respect to $u$ when $s$ is constant (which now has meaning)

Differentiating

$$
\begin{aligned}
& \frac{d E}{d u}=-P_{0} \frac{s}{u^{2}}+\mathrm{mu}+\mathrm{bs}=0 \\
& \frac{d^{2} E}{d u^{2}}=2 P_{0} \frac{s}{u^{3}}+\mathrm{m}>0
\end{aligned}
$$

These equations make sure that there is one unique solution (root) of the derivative, which minimizes the (consumpted) energy since the second derivative of the function $E(s, u)$ is always positive (for $u>0$ ). [Figure 3]

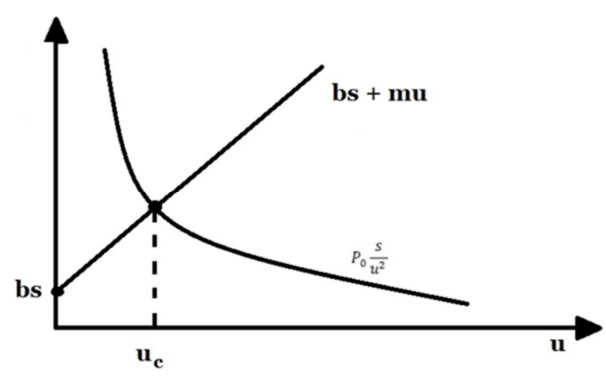

Figure 3. The two components of $\frac{d E}{d u}=0$.

From a physical point of view the situation of the minimization of energy consumption for a certain speed is the following. For large speed, the engine works at a high rate and it consumes a large amount of energy as it passes through a space - interval - $\mathrm{s}$. On the other side, if the vehicle moves at a small speed it consumes low rate energy but for a large time interval (which corresponds to the space s), and finally a lot of energy for the space s. This shows clearly that there exists an indermediate value of $u$ (not very large - not very small) for which we have a minimization of consumpted energy.

It is easy to derive that for a vehicle of the case (1) and for case (2) we have, for a motion that starts with zero speed and easily gets to a constant speed $u_{c}>0$.

$$
\begin{aligned}
& E_{1}\left(\mathrm{~s}, u_{c}\right)=P_{0} \frac{s}{u_{c}}+\frac{1}{2} \mathrm{~m} u_{c}{ }^{2}+\left(\mathrm{b} u_{c}+F_{c g}\right) \mathrm{s} \\
& E_{2}\left(\mathrm{~s}, u_{c}\right)=P_{0} \frac{s}{u_{c}}+\frac{1}{2} \mathrm{~m} u_{c}^{2}+\left(\mathrm{b} u_{c}-F_{g c}\right) \mathrm{s}
\end{aligned}
$$

The differentiation with respect to $u_{c}$ is the same to both eqs. and equally well lead to eqs. (16) and the situation of figure 3 , which is of cource a minimization.

For the case of "arbitrary" $\mathrm{u}(\mathrm{t}) \neq u_{c}$ it is very difficult to handle the eqs. and this will not be attempted by us - at least presently.

From the dynamical eqs., for $u_{c}=$ const, we have

$$
\mathrm{F}=F_{c g}+\mathrm{b} u_{c}, \text { for the case }
$$

and

$$
\mathrm{b} u_{c}=F_{g c}(\mathrm{~F}=0), \text { for the second case }
$$

\section{Revolution Counter - Speed Counter - Equations}

When a car moves, in constant speed or in acceleration or it is going uphill in a certain gear "g" we have for the dynamical equation of motion $[1-4]$ :

$$
\mathrm{m} \frac{d u}{d t}+b_{g} \mathrm{u}+F_{c g, g}=\mathrm{F}(\mathrm{t})
$$

where

$b_{g}$ is the constant of resistance, for the gear "g" and $F_{c g, g}$ is:

$$
F_{c g, g}\left\{\begin{array}{c}
F_{c, g} \text { (horizontal level) } \\
F_{c, g}+m g \sin \alpha_{0}(\text { uphill }) \\
F_{c, g}-m g \sin \alpha_{0}(\text { slightly downhill })
\end{array}\right.
$$

Where

$F_{c g, g}>0$ and the first $\mathrm{g}$ denotes the gravity while the $\mathrm{g}$ after the comma denotes the gear in the gear - box. (for instance $\mathrm{g}=1,2,3, \ldots, 6$ )

From now on, we will be interested, for simplification, only for the horizontal motion and since we are also interested only for the dynamical eqs. keeping the gear constant we can write:

$$
\mathrm{m} \frac{d u}{d t}+\mathrm{bu}+F_{c}=\mathrm{F}(\mathrm{t})
$$

with the engine working in the "idle" work, we can set

$$
\lambda_{0}\left(\frac{d V}{d t}\right)_{0}=\sigma_{0}(r p m)_{0}=\left(\frac{d E}{d t}\right)_{0}=P_{0}
$$

By which it is meant that the consumption of energy has a constant rate $\left(P_{0}\right)$, it is proportional to the frequency of the engine $[(\mathrm{rpm})=$ revolutions per minute $]$ with a constant of proportionality and is also proportional to the rate of (liquid) fuel that enters the cylinders, with constant of proportionality $\lambda_{0}$. The constants of proportionality $\sigma_{0}$ and $\lambda_{0}$, have 
dimensions of energy and energy per unit volume respectively.

For the case of moving with a gear $\mathrm{g}$, we have:

$$
\lambda_{g}\left(\frac{d V}{d t}\right)=\sigma_{g}(r p m)=\left(\frac{d E}{d t}\right)_{g}=P_{0 g}+\mathrm{uF}
$$

Where $g$ stands for gear $g$ and the other symbols have a similar meaning to these of $(21)$ and $\mathrm{u}(\mathrm{t}) \geq 0, \mathrm{~F}(\mathrm{t}) \geq 0$.

Subtracting eqs. (22 and 21) with our attention to the $\sigma$ and $\mathrm{P}$ "componets" we get

$$
\mathrm{uF}=\sigma\left[(r p m)-(r p m)_{0}\right]=\sigma \delta_{r}(\mathrm{t})
$$

where we have also used

$$
P_{0 g}-P_{0} \approx\left(\sigma-\sigma_{0}\right)(r p m)_{0}
$$

If it was not so we should have a force $\mathrm{F}(\mathrm{t}) \neq 0$ (of the engine) even in the case where $\delta_{r}=0$, since a constant term should be added in the right hand side of 23). Besides we expect $P_{0 g}>\approx P_{0}$ and $\sigma_{0 g}>\approx \sigma_{0}$ which is in accordance to the auxiliary equation written above.

Combining eqs. $(20 \& 23)$ we get

$$
\mathrm{m} \frac{d u}{d t}+\mathrm{bu}+F_{c}=\sigma \frac{\delta_{r}(\mathrm{t})}{u}
$$

which is an ordinary differential eqs. (o. d. e.), which if solved gives the speed $\mathrm{u}$ of the car if $\delta_{r}(\mathrm{t})$ is known, provided that also the constants $\mathrm{b}, \mathrm{m}, F_{c} \& \sigma$ are known. (for a gear "g") [1-4]

Since we don't know the function $\delta_{r}(\mathrm{t})$, we have to invent one. Since the rotations (revolutions) of the engine are initially low, then they usually grow quickly (as soon as the throttle is pushed) and finally saturate at a certain level we can suggest:

$$
\delta_{r}(\mathrm{t})=\delta_{r_{i}}+\left(\delta_{r_{f}}-\delta_{r_{i}}\right)\left(1-e^{-\gamma t}\right)
$$

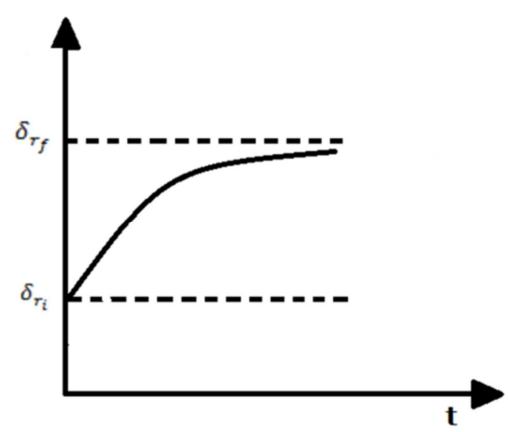

Figure 4. The "saturation" function $\delta_{r}(t)$.

In figure 4 , we have $0<\delta_{r_{i}}<<\delta_{r_{f}}$ where $\delta_{r_{i}}=\delta_{r}(0)$ and $\delta_{r_{f}}=\delta_{r}(+\infty)$

" $\gamma$ " is expressed in $\sec ^{-1}$ and is usually large. At time $\mathrm{t} \simeq$ $5 \gamma^{-1}$, the engine is considered to have "saturated" the revolutions per unit time (no more increase) and almost this time is equivalent to the "infinite time".

All though eq. (25) is "arbitrary" (but it has the features of the revolution counter of a car) its precise form is not important for making results as we shall see later.

We define the quantity $\bar{u}(\mathrm{t})$ such that:

$$
\mathrm{b} \bar{u}+F_{c}=\sigma \frac{\delta_{r}(\mathrm{t})}{\bar{u}}
$$

which is the equation (24) with the first term missing. (i. e. the derivative)

The solution of the last eq. with respect to $\delta_{r}(\mathrm{t})$ is:

$$
\bar{u}=\frac{-F_{C}+\sqrt{F_{c}{ }^{2}+4 \sigma b \delta_{r}(\mathrm{t})}}{2 b}=\frac{2 \sigma \delta_{r}(\mathrm{t})}{F_{c}+\sqrt{{F_{c}}^{2}+4 \sigma b \delta_{r}(\mathrm{t})}}
$$

It is easy to check that in the limit $4 \sigma b \delta_{r}(\mathrm{t})<<F_{c}{ }^{2}$ we get $\mathrm{u}(\mathrm{t}) \simeq \frac{\sigma \delta_{r}(\mathrm{t})}{F_{c}}$ and in the opposite limit $\mathrm{u}(\mathrm{t}) \simeq\left(\frac{\sigma \delta_{r}(\mathrm{t})}{b}\right)^{\frac{1}{2}}$.

From the above information as well as the already known (supposed) function $\delta_{r}(\mathrm{t})$ we can draw a graph of the functions $\bar{u}(\mathrm{t})$ and $\mathrm{u}(\mathrm{t})$.

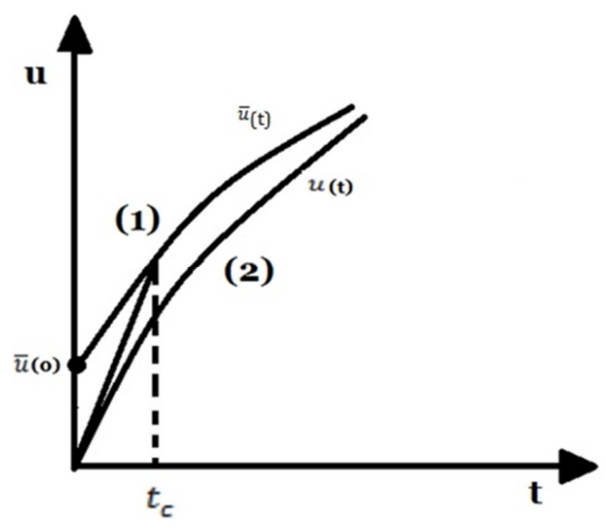

Figure 5. The auxiliary curve (1) and the true curve (2).

Subtracting eqs. (24-26) we get

$$
\mathrm{m} \frac{d u}{d t}=-\mathrm{b}(u-\bar{u})-\sigma \delta_{r}(\mathrm{t}) \frac{u-\bar{u}}{u \bar{u}}
$$

where of course $\bar{u}(\mathrm{t})$ is a known function if the $\mathrm{m}, \mathrm{b}, F_{c}, \sigma$ constants are known (if not known they can be found by suitable observations).

Suppose that for a certain (but random) $t_{0}>0$ we have $0<$ $\mathrm{u}\left(t_{0}\right)<\bar{u}\left(t_{0}\right)$. From the right hand side of (28), we get $\frac{d u}{d t}>0$ and the solution will be increased for the subsequent infinitesimal time.

This will happen continuously and the solution will elevate higher and higher until it will reach a point $t_{c}$ for which $\mathrm{u}\left(t_{c}\right)=\bar{u}\left(t_{c}\right)$. (Figure 5) (Curve 2)

From eq. (28) for $\mathrm{t}=t_{c}$, we have $\frac{d u}{d t}\left(t_{c}\right)=0$, which is a contradiction (line 1) showing definetely that the curve $\mathrm{u}(\mathrm{t})$ cannot intersect the curve $\bar{u}(\mathrm{t})$ at $t_{c}$ horizontally $\dot{u}\left(t_{c}\right)>0$.

Therefore the only possibility left is the "horizontal asymptotic convergence" of the curve $\mathrm{u}(\mathrm{t})$ to $\bar{u}(\mathrm{t})$. (Curves 1 , 2)

To see this better, define $\mathrm{w}=\bar{u}-\mathrm{u}$ and the eqs of motion (28), gives

$$
\mathrm{m} \frac{d w}{d t}=-\left(\mathrm{b}+\frac{\sigma \delta_{r}}{u \bar{u}}\right) \mathrm{w}+\mathrm{m} \frac{d \bar{u}}{d t}
$$

Although we don't know the exact solutions $\mathrm{u}, \bar{u}$ (but only that their positive - definite) as well as b, m, $\sigma, F_{c}, \delta_{r_{i}}, \delta_{r_{f}}$, 
we can prove that $w(\mathrm{t})$ tends asymptotically to zero $(\mathrm{u}(\mathrm{t}) \rightarrow$ $\bar{u}(\mathrm{t}))$ based on the positivity of the term $\left(\mathrm{b}+\frac{\sigma \delta_{r}}{u \bar{u}}\right)$ and the vanishing of the function $\dot{\bar{u}} \simeq 0$ as $\mathrm{t} \gg>\gamma^{-1}$.

Again, eq. (24) has a strange feature the last term becomes infinite if the speed $\mathrm{u} \rightarrow 0^{+}$.

But then, how the solution is initiated when the solution starts from zero speed $(u(0)=0)$ when for instance it is attempted to be solved by a numerical solution?

The paradox is only apparent!

If one performs the transformation $y=u^{2}$ (by which if you know $\mathrm{u}$, you know $y$ ) then one gets the following o.d.e. [1 4]:

$$
\frac{d y}{d t}=\frac{1}{m}\left(2 \sigma \delta_{r}(t)-2 \mathrm{by}-2 F_{c} y^{\frac{1}{2}}\right)
$$

Which can be solved (for instance numerically) even for the initial value problem (i. v. p) for which $y(0)=0 .(u(0)=0)$

\section{Estimation of Physical Constants and Calculation of Car's Power}

So far we have dealt with the o.d.es that describe the car motion assuming, for simplicity, that the motion takes place on the horizontal level, when they are driven by real forces $\mathrm{F}(\mathrm{t})$ or forces phaenomenal which are for instance related mathematically to the mechanical features of the engine (for instance $\left.\delta_{r}(\mathrm{t})\right)[1-4]$.

In both cases, there are constants - parameters that enter the o.d.e(s) phaenomenologically and must be calculated if we want to have an "integrated" picture of the car motion.

As is the construction of the theory there are four constants for every gear where $\mathrm{g}=\{1,2,3, \ldots, 6\}$ for example $b_{g}, F_{c, g}, \sigma_{g}, \lambda_{g}$. The mass of the car is excluded since it is unique for every gear.

The calculation of the $4 \times 6$ constants is useful, for the numerical integration of the eqs. but much more, it is useful for the calculation (theoretical) of the power of the engine (in HP for instance), which if proved correct at least in order of magnitude - is a sign of the correctness of the theory.

[Here by power of the engine, we mean the consumption of fuel energy per unit time at a certain rpm (revolution per minute), in a certain gear and subsequently in a certain speed in the horizontal level].

We are not going to estimate all the $4 \times 6$ constants of the "model" but only these of the $6^{\text {th }}$ gear $\left(b_{6}, F_{c, 6}, \sigma_{6}\right.$ and $\left.\lambda_{6}\right)$ which we will used to calculate the power of the engine in $6^{\text {th }}$ gear at $\mathrm{u}=100 \frac{\mathrm{km}}{\mathrm{h}}(2500 \mathrm{rpm})$ and $\mathrm{u}=120 \frac{\mathrm{km}}{\mathrm{h}}(3000 \mathrm{rpm})$.

If a car is moving in a straight road (horizontal level), initially with speed $u_{0}$, but without use of the gas pedal then it will decelerate from $u_{0}$ to zero.

The o.d.e. describing the motion is

$$
\mathrm{m} \frac{d u}{d t}=-\mathrm{bu}-F_{c}, 0 \leq u(t) \leq u_{0}
$$

The solution of the above o.d.e written in a "peculiar" form is

$$
e^{\frac{b}{m} t}=\left[\frac{u_{0}+\frac{F_{C}}{b}}{u(t)+\frac{F_{C}}{b}}\right]>1
$$

It is important to notice that $0 \leq u(t)$ is equivalent to $0 \leq \mathrm{t}$ $\leq t_{f}$

$$
e^{\frac{b}{m} t} f=\left[\frac{u_{0}+\frac{F_{C}}{b}}{0+\frac{F_{C}}{b}}\right]
$$

For motion in a "straight" road, eq. (24) admits the solution, $\mathrm{u}=$ constant for $\delta_{r}=$ constant.

For two speeds $u_{1}, u_{2}$ and relevant differences of rpms $\delta_{r_{1}}, \delta_{r_{2}}$ we can write

$$
\begin{aligned}
& \mathrm{b} u_{1}^{2}+F_{c} u_{1}=\sigma \delta_{r_{1}} \\
& \mathrm{~b} u_{2}^{2}+F_{c} u_{2}=\sigma \delta_{r_{2}}
\end{aligned}
$$

For instance

$$
\begin{aligned}
& u_{1}=120 \frac{\mathrm{km}}{\mathrm{h}}=33, \overline{33} \frac{\mathrm{m}}{\mathrm{sec}} \\
& u_{2}=100 \frac{\mathrm{km}}{\mathrm{h}}=27, \overline{77} \frac{\mathrm{m}}{\mathrm{sec}}
\end{aligned}
$$

with relevant differences rpm

$$
\begin{gathered}
\delta_{r_{1}}=(3000-900) \mathrm{rpm}=2100 \mathrm{rpm}=35 \mathrm{~Hz} \\
\delta_{r_{2}}=(2500-900) \mathrm{rpm}=1600 \mathrm{rpm}=26, \overline{66} \mathrm{~Hz} \\
\text { And }(r p m)_{0}=900 \mathrm{rpm} \rightarrow \text { “idle” work. }
\end{gathered}
$$

Equations (33) can be written as follows

$$
\begin{aligned}
b^{\prime} u_{1}^{2}+F_{c}{ }^{\prime} u_{1} & =\delta_{r_{1}} \\
b^{\prime} u_{2}^{2}+F_{c}{ }^{\prime} u_{2} & =\delta_{r_{2}}
\end{aligned}
$$

Where

$$
\begin{aligned}
b^{\prime} & =\frac{b}{\sigma} \\
F_{c}{ }^{\prime} & =\frac{F_{c}}{\sigma}
\end{aligned}
$$

Solving for $b^{\prime}, F_{c}{ }^{\prime}$ we get

$$
\left[\begin{array}{l}
b^{\prime} \\
F_{c}{ }^{\prime}
\end{array}\right]=\frac{1}{u_{1} u_{2}\left(u_{1}-u_{2}\right)}\left[\begin{array}{c}
u_{2} \delta_{r_{1}}-u_{1} \delta_{r_{2}} \\
-u_{2}^{2} \delta_{r_{1}}+u_{1}^{2} \delta_{r_{2}}
\end{array}\right]
$$

From which $b^{\prime} \& F_{c}{ }^{\prime}$ may be calculated.

If in the $6^{\text {th }}$ gear, going from $u_{1}$ to speed $u_{2}$ we need $\Delta \mathrm{t} \simeq$ $15 \mathrm{sec}$ for a car of mass $\mathrm{m} \simeq 2000 \mathrm{Kgr}$, we have from (31) and from $b^{\prime}=\frac{b}{\sigma}, F_{c}{ }^{\prime}=\frac{F_{c}}{\sigma}$

$$
\frac{b}{m} \Delta \mathrm{t} \simeq \ln \left[\frac{u_{1}+\frac{F^{\prime}}{b^{\prime}}}{u_{2}+\frac{F \prime}{b^{\prime}}}\right]
$$

The solution of equations (35) give

$$
b^{\prime}=0,0162 \text { (S. I.), }{F_{c}}^{\prime}=0,51001 \text { (S. I.) }
$$

From which 


$$
\begin{gathered}
\left.\frac{F_{c}}{b_{c}}=\frac{F_{c}{ }^{\prime}}{b^{\prime}{ }_{c}}=31.4821 \text { (S. I. }\right) \\
\mathrm{b}=14.472 \frac{\mathrm{kgr}}{\mathrm{sec}}(\mathrm{S} . \mathrm{I} .) \\
\sigma=\frac{b}{b^{\prime}}=893, \overline{33} \text { joule }(\mathrm{S} . \mathrm{I} .) \\
\left(\frac{d E}{d t}\right)_{1}=\sigma(\mathrm{rpm})=\ldots \simeq 60 \mathrm{HP}(3000 \mathrm{rpm}) \\
\left(\frac{d E}{d t}\right)_{2}=\sigma(\mathrm{rpm})=\ldots \simeq 50 \mathrm{HP}(2500 \mathrm{rpm}) \\
F_{c}=\frac{F_{c}{ }^{\prime}}{b^{\prime}{ }_{c}} \mathrm{~b}=31.482 * 14.472 \simeq 45,56 \mathrm{Kp}
\end{gathered}
$$

Our final goal is to give a procedure by which the constants $\lambda_{g}$ (g: gear, $\left.g=1,2, \ldots, 6\right)$ can be calculated.

From the energy - balance equation we have

$$
\left(\frac{d E}{d t}(\mathrm{t})\right)_{g}=\sigma_{g} \operatorname{rpm}(\mathrm{t})=\lambda_{g}\left(\frac{d V}{d t}\right)_{g}
$$

It is clear that the constants $\sigma_{g}$ have dimension of energy while $\lambda_{g}$ have dimension of energy per unit volume of fuel.

The constants $\sigma_{g}$ may be determined by experiments observations like these they took place in the beginning of section 5 .

Integrating the energy - equation one gets

$$
\lambda_{g}=6_{g} \frac{T<r p m>_{T}}{(\Delta V)_{g}}
$$

where:

1. $\sigma_{g}$ is a known constant from the kinematic measurements,

2. $T$ is the time that the engine works in the gear $g$,

3. $(\Delta V)_{g}$ is the volume of the fuel consumed in time $\mathrm{T}$ when the gear - box has the $g^{\text {th }}$ gear and

4. $<r p m>_{T}=\frac{1}{T} \int_{0}^{T} r p m(t) d t$ is the mean value of the revolution counter measurement.

For instance if one measures $2500<\simeq \mathrm{rpm}<\simeq 3000$ he can estimate $\mathrm{rpm} \simeq 2700$ or 2800 for instance.

Knowing all the quantities of the right hand side, one can easily calculate $\lambda_{g}(g=1,2, \ldots, 6)$. Similarly to eq. (37) one can write for the idle work

$$
\lambda_{0}=\sigma_{0} \frac{T(r p m)_{0}}{(\Delta V)_{0}}
$$

where:

1. $(\mathrm{rpm})_{0}=$ revolutions of the idle work

2. $T$ =time of working engine in the "idle" work

3. $\Delta V=$ volume of fuel used by the engine at time $\mathrm{T}$ (when there is no gear in the gear - box and no gas petal is pressed)

Now, unfortunately the constance $\lambda_{0}, \sigma_{0}$ can not be measured by kinematic mesurements and their calculation needs the extension to a theory of the engine.

One final remark concerns the mass $(\mathrm{m})$ of the car which was probably overestimated the $\mathrm{m}=2000 \mathrm{Kgr}$. If instead we consider $\mathrm{m}=1500 \mathrm{Kgr}$ which is most likely, then for the same "kinematic measurements" we find that $\sigma \rightarrow \frac{1500}{2000}$ $\sigma=\frac{3}{4} \sigma$

From which we get

$$
\left(\frac{d E}{d t}\right)_{1} \rightarrow 45 \mathrm{HP} \text { and }\left(\frac{d E}{d t}\right)_{2} \rightarrow 37,5 \mathrm{HP}
$$

Calculations that seem to be more natural in order of magnitude for the high speed $\left(120 \& 100 \frac{\mathrm{Km}}{\mathrm{h}}\right)$ of the car, in the $6^{\text {th }}$ gear and the smaller mass of the car.

*[TOYOTA (YARIS), 2016, 1330cc]

\section{Conclusions}

As we saw in the main text, if one uses Newton's second law and the tangential forces exerted on the car, such as the tangential force of the engine (which also determines part of the consumpted energy), the friction - air resistance and the component of the weight tangential to the velocity, one can predict the velocity of the vehicle and compare it to this "taken" from the tachograph (speedometer) and the rotation counter (rpm). The true solution will be taken from an o.d.e. which has no "analytical" solution but can be solved numerically if some parameters such as $\mathrm{b}, F_{c}, \mathrm{~F}(\mathrm{t})$, [and $\sigma, \lambda$ (for energy considerations)], are known.

Finally from the motion of a car with constant speed at a straight and flat road (which means no acceleration, no uphill going) and from the registers of the tachograph and the rotation - counter, the parameter $F_{c, 6}, b_{6}, \sigma_{6}, \lambda_{6}$ can be evaluated. Where 6 refers to the $6^{\text {th }}$ gear.

These parameters can be used for the numerical integration of the initial o.d.e. to examine consistency. (This is not done by us).

Finally for motion in a flat - straight road the overall consumed power can be calculated and it is found to be in very reasonable measures in order of magnitude (i.e. $45 \mathrm{HP}$ for $\mathrm{u} \simeq 120 \frac{\mathrm{km}}{\mathrm{h}}, \mathrm{rpm} \simeq 3000 \mathrm{rpm}$ in the $6^{\text {th }}$ gear).

\section{Acknowledgements}

I would like to thank in advance miss Katerina Giannelou for preparing the manuscript.

\section{References}

[1] E. L. Ince, Ordinary Differential Eqs, dover publications 1958.

[2] J. K. Hale, Ordinary Differential Eqs - Wiley Interscience London 1971.

[3] S. Trahanas, Ordinary Differential Eqs - Cretan University Press. Heraklion - Crete -Greece 1989.

[4] Lawrence Perko, Differential Eqs and Dynamical Systems, Springer 2000.

[5] The physics of racing: Weight transfer 2016, Allen Bery.

[6] Motion \& Forces : Newton's Second Law of motion / Perkins.

[7] Newton's Laws of Motion \& Car Physics, Carly Hallman. 
[8] Car Basics - Sir Isaac Newton's Laws of Motion Quinton Kenrick.

[9] The science Behind safe driving.

[10] Newton's Second Law - University Physics (Volume 1)

[11] Car Physics and Newton's Laws of Motion - Effects.

[12] Seat Belts \& Newton's Second Law of Motion - Chris Deziel.

[13] M. G. Calkin, "The motion of an accelerating automobile," Am. J. Phys. 58, 573-575 (June 1990).

[14] R. G. Carson, "Everyday physics: Auto test report," Phys. Teach. 21, 369-375 (Sept. 1983).

[15] J. Güémez and M. Fiolhais, "Forces on wheels and fuel consumption in cars," Eur. J. Phys. 34, 1005-1013 (2013).
[16] J-P Meyn, "The kinematic advantage of electric cars," Eur. J. Phys. 36, 065037 (2015).

[17] R. Cross, "Rolling to a stop down an inclined plane," Eur. J. Phys. 36, 065047 (2015).

[18] B. A. Sherwood, "Pseudowork and real work," Am. J. Phys. 51, 597-602 (July 1983).

[19] D. Tabor, "The rolling and skidding of automobile tyres," Phys. Educ. 29, 301-306 (1994).

[20] C. M Haaland, "Minimum engine size for optimum automobile acceleration," Am. J. Phys. 60, 415-422 (May 1992). 